\title{
Penetrating keratoplasty for keratoconus: complications and long-term success
}

Corneoplastic Unit, Queen Victoria Hospital, East Grinstead K W Sharif T A Casey

Correspondence to: Mr Khaled W Sharif, FRCS, Corneo-Plastic Unit, Queen, Vorneo-Plastic Unit, Qu Gictoria Hospital, East GDZ.

Accepted for publication 6 September 1990

\begin{abstract}
A series of 100 penetrating keratoplasties for keratoconus performed between 1968 and 1986 were reviewed for long-term results. The mean follow-up was 6.1 years with a range of 4-16 years. The systemic associations and the postoperative complications were analysed. Grafting in cases associated with Down's syndrome had a higher incidence of complications. $93 \%$ of grafts remained clear and $81 \%$ achieved a final corrected visual acuity of $6 / 12$ or better. $21 \%$ of eyes developed a homograft reaction, with $50 \%$ of rejection episodes occurring in the first year after operation. Factors associated with higher incidence of rejection included loose sutures, traumatic wound dehiscence, and grafts larger than 8.5 $\mathrm{mm}$. Only three grafts with rejection episodes lost graft clarity, while rejection in the rest was successfully reversed with topical steroid therapy. No relationship was found between donor age and long-term graft clarity.
\end{abstract}

Keratoconus patients have one of the highest success rates of any group of patients undergoing penetrating keratoplasty. Since Castroveijo reported successful grafting for keratoconus in 1927, many reports have confirmed a favourable prognosis. ${ }^{1-4}$ Many of the published reports, however, had a limited follow-up time, and the life span of some corneal grafts has been questioned after specular microscopic studies that showed a continuous decline in the endothelial cell count after keratoplasty. ${ }^{5}$

Although graft clarity can be expected in more than $90 \%$ of cases, postoperative complications in the form of high astigmatism, posterior subcapsular cataract, and secondary glaucoma can lead to a less than perfect visual outcome.

The aim of our study was to select a group of keratoconus patients previously unoperated upon and determine the long-term graft clarity, the relationship of the donor age to graft clarity, the incidence of operative and postoperative complications, including homograft rejection, and finally to determine the probability of graft survival in the group of patients who developed one or more rejection episodes during the followup period.

\section{Materials and methods}

The subjects of the present study were keratoconus patients who underwent penetrating keratoplasty over an 18-year period at the CorneoPlastic Unit, Queen Victoria Hospital. All the patients undergoing this procedure were included provided they had had no previous intraocular surgery and had a four-year minimum follow-up time. One hundred penetrating keratoplasties met these criteria. The case notes were reviewed, and follow-up information was sought from the referring ophthalmologist in nine cases that had not been evaluated in our clinic during the past two years. Some of these patients selected for our study had regrafts or other surgical procedures after the initial keratoplasty. However, our graft success results relate to the original keratoplasty.

Despite the fact that in most cases when a failure occurred a subsequent graft was successfully performed, we have included these cases in our statistics as 'failed grafts', since our aim was to define the prognosis of the initial surgery.

All the patients in our series were selected for surgery if they could not obtain industrially useful vision $(<6 / 18)$ with contact lenses or spectacles, or if they could obtain improved vision with contact lenses but only for limited periods of time. Donor tissues was obtained from whole globes stored at $4^{\circ} \mathrm{C}$ for up to 24 hours with the grafts performed before 1984 (86 grafts). Corneae stored in McCarey-Kaufman medium and chondroitin sulphate dextran (K-sol) were used in the subsequent 14 grafts (1984-6).

Penetrating keratoplasties in our series were performed according to the method in use during that time interval, and the numbers per period are indicated in Table 1. All the operations were performed under general anaesthesia by the senior author of this report (TAC) and the fellows of the Corneal Unit. Owing to the change in the method of tissue storage since 1984 all the donor corneae were trephined from the endothelial side by a punch trephine. Before 1975 grafts were sutured with interrupted 8-0 virgin silk (29 cases). From $197510-0$ nylon sutures (interrupted and continuous) were used instead (71 cases). At the conclusion of surgery dexamethasone $4 \mathrm{mg}$ and gentamicin $20 \mathrm{mg}$ were injected subconjunctivally and Chloramphenicol $1 \%$ eyedrops were instilled. Postoperatively Chloramphenicol $1 \%$ eyedrops and dexamethasone $0.1 \%$ eyedrops were used routinely four times a day for one month, after which they were tapered off gradually.

The results reported were based on the appearance of the graft at the last clinical examination and the patient's visual acuity. At the same time the hospital notes were reviewed to determine any interim postoperative compli-

Table 1 Surgical techniques

\begin{tabular}{llcl}
\hline Date & Donor trephine & Suture type & $\begin{array}{l}\text { Number of } \\
\text { grafts }\end{array}$ \\
\hline $1968-75$ & Epithelial side & $8-0$ silk & 29 \\
$1975-84$ & Epithelial side & $10-0$ nylon & 57 \\
$1984-6$ & Endothelial side & $10-0$ nylon & 14 \\
\hline
\end{tabular}


cations. Grafts with total or partial oedema were counted as failed or failing.

Graft rejection was diagnosed by the appearance in a previously clear graft of at least one of the following: (1) endothelial rejection line; (2) keratic precipitates; or (3) localised graft oedema, the edge of which usually coincided with the keratic precipitates.

Graft survival has been expressed graphically by Kaplan and Meier survival curves, which take account of staggered recruitment and variable follow-up such that many grafts remained clear at final review and were denoted censored data. They were censored because eventual failure cannot be excluded, but the event has not been observed.

When a rejection was diagnosed, the therapy consisted of topical steroids ever two hours and topical prophylactic antibiotics four times a day along with subconjunctival dexamethasone injections according to the patient's response.

\section{Results}

One hundred penetrating keratoplasties for keratoconus have been examined retrospectively. The grafts in our series had an average follow-up of $6 \cdot 1$ years with a range of 4-16 years. The following indices were recorded:

1. Age and sex. Patients ranged in the age at the time of diagnosis from six to 48 years, mean $9 \cdot 2$ years. Age at the time of penetrating keratoplasty ranged from 11 to 51 years, mean 27.9 years. There were 68 males and 32 females in our series (2:1 ratio).

2. Systemic and ocular associations. The various associations of keratoconus in our patients are listed in Table 2; manifestations of atopy were the most common. Keratoplasties were performed in four cases of Down's syndrome when advanced keratoconus had led to repeated attacks of acute hydrops.

3. Early and late complications. Herniation of the iris-lens diaphragm occurred during $10 \%$ of the keratoplasties (Fig 1), but this was treated easily at the time with no effect on the final outcome. One patient had a $2 \mathrm{~mm}$ hyphaema following a peripheral iridectomy, which resolved spontaneously by the fourth postoperative day.

The paretic pupil, resulting from iris sphincter paralysis, occurred in $4 \%$ of the cases. In all of these cases a fixed dilated pupil was noted on the first postoperative day and it remained dilated over the follow-up period (Fig 2). All these cases were given Pilocarpine $2 \%$ eyedrops in four doses one hour preoperatively with no mydriatics instilled postoperatively. None of these cases had a wound leak with flat anterior chamber, and

Table 2 Systemic and ocular associations

\begin{tabular}{lc}
\hline Association & Percentage of cases \\
\hline I. Systemic & 13 \\
Hay fever & 8 \\
Asthma & 7 \\
Eczema & 4 \\
Down's syndrome & 1 \\
Osteogenesis imperfecta & 2 \\
II. Ocular & 1 \\
Vernal keratoconjunctivitis & Retinitis pigmentosa \\
\hline
\end{tabular}

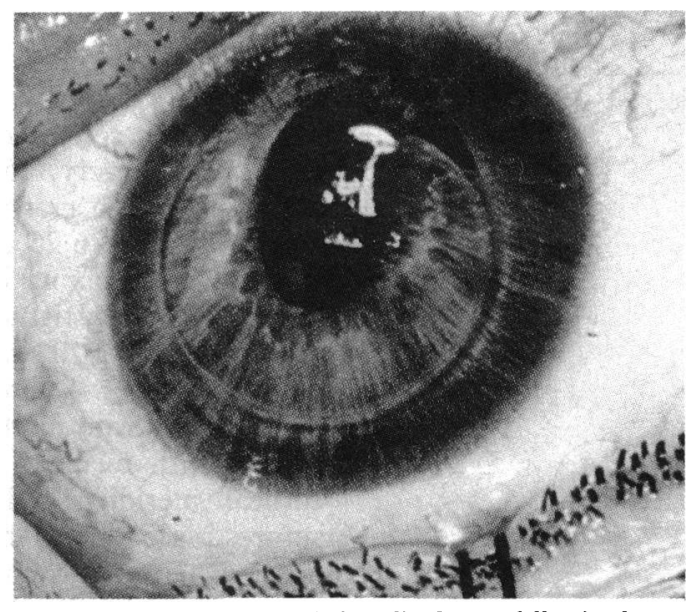

Figure 1 Bulging of the iris-lens diaphragm following host corneal trephination.

there were no anterior synechiae. Only one -patient had associated secondary glaucoma. After the routine use of intravenous mannitol preoperatively in 1976 only one case of a paretic pupil was reported in the subsequent 68 grafts.

Cataract. Posterior subcapsular cataract developed in $12 \%$ of our cases. The clinical onset varied from 9 months to three years postoperatively (average 18 months). The onset and progression of these cataracts were directly related to the duration of topical steroid therapy. This is clearly demonstrated by the fact that $58 \%$ of the patients who developed cataract had previously been treated for one or more rejection episodes, calling for more intensive and prolonged topical steroid therapy than the routine postoperative regimen. Cataract extraction, however, was indicated in only $5 \%$ of the patients in our series, - that is $41.6 \%$ of those who developed cataracts.

Secondary glaucoma. This occurred in $4 \%$ of our cases. One of them was successfully treated by topical therapy, while the remaining three required surgical management. The cases requiring surgery to control the raised pressure all had previous history of rejection episodes involving prolonged topical steroid therapy.

Keratitis. Three cases in our series developed infective keratitis. Two were bacterial keratitis in patients with Down's syndrome, and the third was a fungal keratitis in an immune-compromised eye due to intensive steroid treatment for a rejection episode.

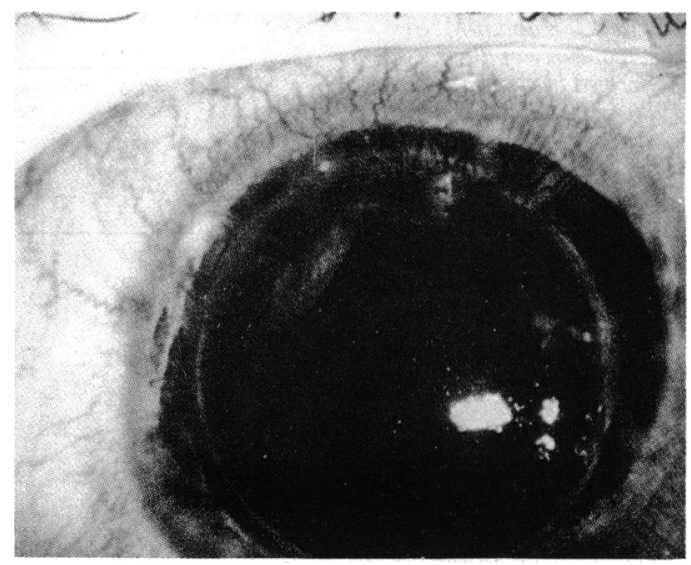

Figure 2 Dilated, fixed pupil following keratoplasy for keratoconus. 
Astigmatism. Astigmatism that could not be successfully neutralised with glasses or contact lenses, following the removal of sutures, occurred in $8 \%$ of the cases. All of them were treated with refractive surgery. $5 \%$ of the cases had astigmatism with a range of 5.5-8.0 dioptres and a mean value of 6.25 dioptres. This group was treated with relaxing incisions with successful reduction of the astigmatism to a mean value of 2 dioptres, leading to successful visual rehabilitation with glasses. The remaining three cases with high astigmatism, more than 8.5 dioptres, were treated with relaxing incisions at the steepest corneal meridian followed by augmentation sutures placed along the flattest meridian. This method helped to reduce the astigmatism to a mean value of $2 \cdot 25$ dioptres in two cases. The third case had a residual astigmatism of 5.5 dioptres after this method which was treated with wedge resection, leading to successful visual rehabilitation with glasses.

Graft rejection. Homograft rejection occurred in $21 \%$ of our cases. Only one of these was an epithelial rejection, which developed seven months postoperatively.

The mean time to the endothelial rejection episodes was 1.8 years, range 17 days to 15 years (Fig 3). Five of these cases had a second rejection episode during the follow-up. Thirteen of the rejection episodes (50\%) occurred in the first 12

Figure 3 Onset of homograft rejection in relation to postoperative follow-up time.

Figure 4 Kaplan-Meier survival curve of all grafts performed between 1968 and 1986 for keratoconus.

Figure 5 Comparison of graft survival in groups $A$ and $B$.
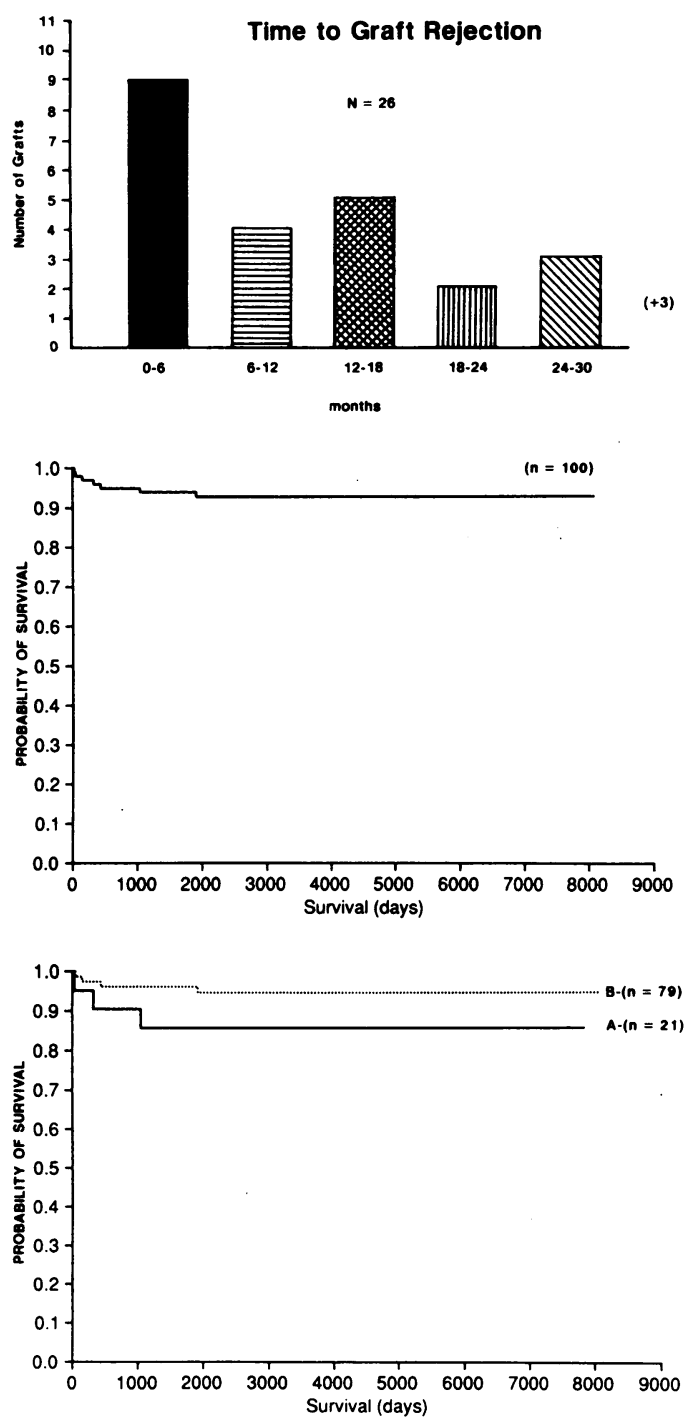

months. Seven eyes (26.9\%) developed a rejection in the second year. Five of the remaining six patients who had a rejection after the second year had had a previous rejection earlier. Factors associated with these rejection episodes included loose sutures in six eyes, one following a traumatic wound dehiscence, and one following treated infective keratitis. The probability of graft survival for all penetrating keratoplasties performed in one series between 1968 and 1986 $(n=100)$ was $93 \%$ at 2500 days $(6 \cdot 8$ years) (Fig 4). Most failures occurred in the first 500 days (1.37 years). The survival curves in (Fig 5) compare the probability of survival between the group of patients wtih one or more rejections episodes (group A) and those with no history of rejection (group B). It is clearly shown that the probability of survival of group B was $96 \%$ at 1000 days $(2 \cdot 7$ years), while that of group A was $86 \%$ at the same time. We examined the incidence of the graft rejection in relation to the donor size (Table 3). Variation of the graft size between 7 $\mathrm{mm}$ and $8.5 \mathrm{~mm}$ had no statistically significant effect on the rejection rate. However, $9 \mathrm{~mm}$ grafts had a high rejection incidence $(50 \%)$.

All but three rejection episodes were successfully reversed with topical steroid therapy. The period between the onset of the rejection and the return of the graft varied from 17 days to 140 days, mean 47 days.

4. Long-term graft clarity. $96 \%$ of the grafts in our series were clear by the end of the first postoperative year, and $93 \%$ were still clear by the end of an average follow-up of $6 \cdot 1$ years. Only seven grafts failed in our series. The cause of failure are listed in Table 4. In a subgroup of ten eyes with an average follow-up of 15 years, $90 \%$ of the grafts remained clear.

5. Donor age and graft clarity. Donor age was available for 93 of the 100 grafts $(93 \%)$. The mean follow-up of these grafts was $8 \cdot 1$ years. The donor age ranged from 13 years to 91 years, mean 65.5 years. Donors were divided into three groups to detect any relationship between longterm graft clarity and donor age, and these groups were: donor age $<45,45-65$, and $>65$ (Table 5). We could not find any statistically

Table 3 Rejection incidence in relation to graft size

\begin{tabular}{lll}
\hline & \multicolumn{2}{l}{ Rejection } \\
\cline { 2 - 3 } Size $(\mathrm{mm})$ & \% Proportion \\
\hline $7 \cdot 0$ & $16 \cdot 6$ & 1 of 6 \\
$7 \cdot 5$ & $2 \cdot 3$ & of 13 \\
$8 \cdot 0$ & $16 \cdot 6$ & 12 of 72 \\
$8 \cdot 5$ & 25 & 1 of 4 \\
$9 \cdot 0$ & 50 & 3 of 6 \\
\hline
\end{tabular}

Table 4 Causes of graft failure

\begin{tabular}{ll}
\hline Causes & Number of grafts \\
\hline Graft rejection & 3 \\
Late graft decompensation & 2 \\
Infective keratitis & 2 \\
\hline
\end{tabular}

Table 5 Donor age and graft clarity (93 grafts)

\begin{tabular}{lccl}
\hline Donor age & Total number & Clear & Failed \\
\hline Less than 45 years & 7 & 6 & 1 \\
45-65 years & 34 & 32 & 2 \\
More than 65 years & 52 & 49 & 3 \\
\hline
\end{tabular}


Table 6 Long term visual acuity

\begin{tabular}{lc}
\hline Visual acuity & \% of eyes \\
\hline $6 / 9$ or better & 59 \\
$6 / 12$ & 22 \\
$6 / 18$ & 7 \\
$6 / 24-6 / 60$ & 8 \\
Less than $6 / 60$ & 4 \\
\hline
\end{tabular}

Table 7 Causes of visual acuity less than 6/36

\begin{tabular}{ll}
\hline Causes & \% of eyes \\
\hline Failed graft & 3 \\
Secondary glaucoma & 2 \\
Retinal disease & 2 \\
Healed infective keratitis & 1 \\
\hline
\end{tabular}

significant relationship between long-term graft clarity and donor age $(p=0 \cdot 38)$, which was consistent with previously published reports. ${ }^{6}$

6. Long-term visual outcome. The final corrected visual results of the grafts in our series over an average follow-up of $6 \cdot 1$ years are listed in Table $6.81 \%$ of the eyes achieved visual acuity of $6 / 12$ or better. Residual high astigmatism, decompensation of the graft, and steroid induced cataract were the major cause of decreased acuity. The causes of visual acuity of $6 / 60$ or less are listed in Table 7. The majority of these eyes were successfully regrafted and had a good visual result.

\section{Discussion}

Keratoconus is a non-inflammatory axial ectasia of the cornea with an estimated incidence of 1:20 000. It has a slowly progressive course resulting in high degrees of irregular, myopic astigmatism. Although some reports have shown the condition to be predominant in females, ${ }^{7}$ our series had a predominance of males $(68 \%)$. This is in agreement with reports by Buxton ${ }^{8}$ and Woodward, ${ }^{9}$ who found males to be predominant with proportions of $62 \%$ and $60 \%$ of their cases respectively.

Various systemic and ocular associations have been found in our case (Table 2). Association with atopy, eczema, and vernal conjunctivitis is important, since these patients may have more immunological problems than expected in nonvascularised corneae. ${ }^{10} 11$ The most advanced cases of keratoconus seen in our series were those associated with Down's syndrome (four cases). The mental retardation of these patients always carries the risk of trauma or infection of an otherwise successful graft. Infective keratitis led to the failure of the initial graft in two of these four cases. The decision to operate on such patients should be based on individual assessment of each case, with particular consideration of the degree of visual impairment, the grade of mental retardation, and the presence or absence of adequate post operative home care and supervision.

The complications following penetrating keratoplasty for keratoconus are relatively few compared with those encountered in grafting high risk patients. The development of a dilated fixed pupil as a complication of grafting in keratoconus has been noted by a number of surgeons including Castroveijo and Paufique, but it was Urrets-Zavalia ${ }^{12}$ who first published his observations and suggested that the association of fixed dilated pupil, iris atrophy, and secondary glaucoma constituted a specific syndrome which has since been frequently known by his name. In our series $4 \%$ of the cases had paretic pupils from the first postoperative day. However, only one developed secondary glaucoma, which confirms previously published reports by Davies and Ruben that glaucoma is not a sequel to the simple paretic pupil. ${ }^{13}$ Glaucoma thus seems to be no more a special complication of keratoplasty in keratoconus than it is of keratoplasty for any other corneal lesion. The paretic pupil can be explained on the basis of ischaemic atrophy of the sphincter pupillae muscle secondary to an iris strangulation phenomenon occurring as a result of anterior displacement of the iris lens diaphragm against the posterior surface and cut edge of the host cornea during surgery. This theory was confirmed in our experience by the fact that, since preoperative intravenous mannitol $20 \%$ was used routinely for all grafts performed after 1976, there has been a significant decrease in the incidence of this complication. One case out of 68 grafts had a paretic pupil compared with three out of 32 grafts performed before 1976.

Posterior subcapsular cataract developed in $12 \%$ of our patients. However, less than half of these cataracts $(41 \cdot 6 \%)$ required extraction. The relation between long-term topical steroid use and the development of cataract and secondary glaucoma was highlighted by the fact that the majority of eyes having these complications in our series were those that required intensive and more prolonged steroid therapy for the treatment of rejection episodes.

There have been variable reports on the incidence of homograft reaction following keratoplasty for keratoconus. Chandler and Kaufmann reported a high incidence of $35 \%$ in 1974. ${ }^{14}$ A recent report by Trautman and Lambers ${ }^{15}$ gave a lower incidence of $11 \cdot 6 \%$. Our long-term follow-up study (1968-90) showed the incidence to be $21 \%$. The variations of rejection rate among different reports are most probably attributed to the changes in surgical techniques at various times, with the advent of the 10-0 nylon suture closure replacing the previously used virgin silk.

Most of the graft rejections in our study were successfully treated with topical and subconjunctival steroids, and only $3 \%$ of the eyes lost graft clarity as a result of endothelial rejection. It was interesting to find that $50 \%$ of rejection episodes occurred during the first twelve months post operatively - that is, before suture removal - and that nearly all the eyes that developed a rejection after the second year had a history of an earlier rejection that has been reversed. No significant correlation could be made between the graft size and the risk of rejection when donor size was between 7.0 and $8.5 \mathrm{~mm}$ (Table 3 ). It was interesting, however, to note that larger grafts, namely $9.0 \mathrm{~mm}$, had a higher incidence of rejection reactions $(50 \%)$. This is probably related to the greater proximity to the limbus and the larger antigenic load.

The long-term results in our series confirm the excellent prognosis found in several previous 
reports $^{15-17} 93 \%$ of our cases maintained graft clarity over an average follow-up period of $6 \cdot 1$ years. The excellent rate of graft clarity in the subgroup with a 15 -year average follow-up (90\%) indicates that the progressive endothelial cell loss reported with specular microscopy over the first year postoperativelys does not forecast ultimate graft failure.

As indicated in Table $681 \%$ of penetrating keratoplasties provided $6 / 12$ or better vision after 6.1 years average follow-up. $40 \%$ of the cases required spectacle correction to achieve this acuity compared with $26 \%$ requiring contact lenses and $15 \%$ needed no correction.

In summary, penetrating keratoplasty at the present time appears to be the procedure of choice in treating keratoconus, with excellent long-term visual results. The few postoperative complications can be minimised if the patient has been subject to careful follow-up and has been instructed to report immediately if pain, redness, or visual loss develops. The incidence of iatrogenic cataract and secondary glaucoma can be reduced by avoiding the liberal use of topical steroids and by rapidly tapering them off in complicated cases. It is also better to avoid a graft size larger than $8.5 \mathrm{~mm}$, as this carries a higher incidence of rejection reactions.
We thank Mrs Beth Chase-Grey, Mrs Margot Bain, and Mrs Alma Ayles for their great assistance in the preparation of this manuscript. We are grateful to Dr I D Hill for his valuable assistance in the medical statistics of the paper.

1 Castroveijo R. Surgery of the Cornea. Int Abstr Surg 1937; 65: 489-505.

2 Castroveijo R. Keratoplasty in treatment of keratoconus. Arch Ophthalmol 1949; 42: 776-800.

3 Ehlers $\mathbf{N}$, Olsen T. Long term results of corneal grafting in keratoconus. Arch Ophthalmol (Kbh) 1983; 61;918-26.

4 Keates RH, Falkenstein S. Keratoplasty in keratoconus. $A m \mathcal{F}$ Ophthamol 1972; 74: 442-4.

5 Bourne WM. One year observation of transplanted human corneal endothelium. Ophthalmology 1982; 89: 600-4.

6 Forester RK, Fine M. Relation of donor age to success in penetrating keratoplasty. Arch Ophthalmol 1971; 85: 42-7.

7 Leibowitz HM. Comeal disorders, clinical diagnosis and management. Ist ed. Philadelephia: Saunders, 1984: 100-20.

8 Buxton JN. Keratoconus in symposium on contact lenses. Trans New Orleans Acad Ophthalmol 1973; 3: 88-100.

9 Woodward EG. Keratoconus: epidemiology. $\mathcal{F}$ Br Contact Lens Assoc 1984; 7: 64-76.

10 Copeman PWM. Eczema and keratoconus. Br Med $\mathcal{F} 1965$; ii: 977-8.

11 Spincer WH, Fisher JJ. The association of keratoconus with atopic dermatitis. Am 7 Ophthalmol 1959; 47: 322-4.

12 Urrets-Zavalia A. Fixed, dilated pupil, iris atrophy and secondary glaucoma. Am f Ophthalmol 1963; 56: 257-65.

13 Davies PD, Ruben M. The paretic pupil: its incidence and aetiology after keratoplasty for keratoconus. Br $\mathcal{J}$ Ophthalmol 1975; 59: 223-8.

14 Chandler JW, Kaufmann HF. Graft reactions after keratoplasty for keratoconus. Am $\mathcal{F}$ Ophthalmol 1974; 77: 543-7.

15 Trautman RC, Lambers MA. Penetrating keratoplasty for keratoconus. Cornea 1987; 6: 289-305.

16 Abbot RL, Forester RK. Determinant of graft clarity in penetrating keratoplasty. Arch Ophthalmol 1979; 97: 10715.

17 Paglen PG, Fine M, Abbot RL, Webster RG. The prognosis for keratoplasty in keratoconus. Ophthalmology 1982; 89: $651-4$.
Although there is much further work to be done on the subject, from the limited number of cases treated we think the following conclusions may be drawn:

1. In certain corneal conditions - notably ulceration, superficial type of keratitis and chronic corneal opacities - treatment by means of ascorbic acid intravenously is of therapeutic value. The improvement in most cases is almost dramatic.

2. In most cases treated, there is no reason to believe that a general vitamin ' $C$ ' deficiency exists. It appears, therefore, that the beneficial results are obtained by flooding the bloodstream with excess of ascorbic acid.

3. Ascorbic acid appears to be of no value in the treatment of iritis. It may even have a detrimental effect in certain instances.

4. The injections used (ascorbic acid 'Roche' concentrated vitamin ' $C$ ' ampoules, $500 \mathrm{mgs}$ per injection) caused no local or general reaction.

5. Dosage: We recommend the daily injection of $500 \mathrm{mgs}$ intravenously until active inflammation of the eye has ceased, preferably followed by ascorbic acid tablets given orally (Tabs. ii.t.d.s., each tablet containing $250 \mathrm{mgms}$ ). - Conclusion of an article by Wing-Commander Keith Lyle T, Squadron-Leader McLean DW. Vitamin C (ascorbic acid) - its therapeutic value in inflammatory conditions of the cornea. Brf Ophthalmol 1941; 25: 286-95. 